\title{
La formación en materias de tecnología del futuro periodista en España
}

\section{The training in technology subjects of the future journalist in Spain}

Javier Sierra Sánchez. Universidad Complutense de Madrid. España. javiersierrasanchez@pdi.ucm.es

$[\underline{\mathrm{CV}}]$ (8) $\mathrm{G}$

Sheila Liberal Ormaechea. Universidad Francisco de Vitoria. España.

s.liberal.prof@ufv.es

$[\mathrm{CV}]$ (8)

Luis Mañas-Viniegra. Universidad Complutense de Madrid. España. $\underline{\text { lmanas@ucm.es }}$

$[\mathrm{CV}]$ (c) $\mathrm{G}$

Este trabajo se ha realizado mediante la financiación del proyecto "Conductas y competencias sociocomunicativas de la infancia y la juventud en Tecnologías de la Información y Comunicación (TIC)" de la UCM.

Cómo citar este artículo / Referencia normalizada

Sierra Sánchez, J., Liberal Ormaechea, S. y Mañas-Viniegra, L. (2020). La formación en materias de tecnología del futuro periodista en España. Revista Latina de Comunicación Social, 75, 189206.

https://www.doi.org/10.4185/RLCS-2020-1422

\section{RESUMEN}

Introducción. En este trabajo de investigación empírica analizamos la introducción de asignaturas en materia tecnológica que ofrecen las universidades españolas (públicas y privadas) a través de los Grados de Periodismo/Comunicación. Metodología. En el artículo analizamos, mediante la técnica de análisis de contenido, treinta y ocho planes de estudio de Grado a partir de un universo de más de mil novecientas asignaturas. Resultados. La investigación descubre hallazgos interesantes en cuanto al grado de implementación, descubriendo qué tecnologías emergentes están reflejándose en los diseños de los planes de estudio actuales en España. Conclusiones. Se indican cuáles son las universidades españolas que más carga crediticia y número de asignaturas ofrecen relacionadas con la tecnología.

PALABRAS CLAVE: tecnologías; Comunicación; contenidos digitales; planes de estudio.

\begin{abstract}
Introduction. In this empirical research we analyse the introduction of technology subjects offered by Spanish universities (public and private) through the Journalism / Communication graduate studies. Methodology. In the article we analyse, through the technique of content analysis, thirtyeight degree study plans out of a universe of more than one thousand nine hundred subjects. Results. The research discloses interesting findings, like implementations and emerging technologies are
\end{abstract}


being reflected in the designs of current curricula in Spain. Conclusions. We also indicate which are the Spanish universities with the highest credit load and number of subjects offered in terms of technology.

KEYWORDS: technologies; Communication; digital content; study plans.

\section{CONTENIDO}

1. Introducción. 2. La necesaria formación en tecnologías para los futuros periodistas 3. Objeto, Hipótesis y Objetivos de la Investigación. 4. Metodología de investigación. 5. Resultados de la investigación: descripción y análisis 6. Conclusiones. 7. Referencias bibliográficas.

Traducido por Yuhanny Henares (Traductora académica, Universitat de Barcelona).

\section{Introducción. La importancia de la formación en Tecnología para ejercer el Periodismo actual}

Este trabajo empírico pretende complementar las investigaciones realizadas por Sánchez-García, Campos-Domínguez y Marinho (2016) sobre la formación digital de los programas de Periodismo, estableciendo una comparativa entre España y Portugal.

Actualmente, formamos parte de un ecosistema digital en el que se están dando interacciones complejas entre cultura, economía y tecnología en un mundo globalizado que es dominado por símbolos, textos, sonidos e imágenes. En esta vertiginosa ola de cambio, se están redefiniendo las bases de la innovación y la productividad, pero también los modelos y paradigmas del periodismo clásico y la forma de empleo futuro.

Diferentes investigaciones han profundizado en esa transición desde el periodismo tradicional al periodismo digital, poniendo el foco en diferentes aspectos como la convergencia (Salaverría y García Avilés, 2008), la virtualización tecnológica (Fondevila, 2010) o las destrezas y perfiles profesionales (Meso Ayerdi, 2003; Scolari, Micó y Navarro, 2008; López García, 2010; Sierra y Cabezuelo, 2009; Marinho, 2012 y Rosique, 2013; Jiménez-Gómez y Mañas-Viniegra, 2018).

La eclosión de las TIC y de otras tecnologías emergentes, así como el desarrollo de los contenidos digitales, están modificando la forma de hacer periodismo. Podemos observar cómo la realidad virtual ha generado lo que algunos expertos denominan periodismo inmersivo, provocando que el espectador se introduzca por completo en la historia. Por otro lado, el big data juega un papel clave en el periodismo de investigación y la inteligencia artificial está siendo crucial en la automatización de rutinas periodísticas. De estas innovaciones que afectan a las funciones que ejerce un periodista en una redacción se ha hecho eco Lerma Noriega (2018, p. 1441) al afirmar que "las funciones clásicas del periodista han variado, especialmente en aquellos aspectos que tienen que ver con la habilidad multimedia, pues se han gestado nuevos perfiles profesionales: diseñadores, animadores, infógrafos, analistas en big data, desarrolladores web, desarrolladores de APP, etc.".

En esta misma línea, Salaverría y García Avilés (2008, p. 43) advierten sobre cómo la tecnología incrementa la productividad, ya que al redactor no sólo se le exige que sepa escribir, sino que también otros conocimientos como editar vídeos, maquetar, publicar en web, aspectos básicos de fotografía y fundamentales de locución, muy en consonancia con lo que será el perfil del periodista multimedia. 
Según la consultora Gartner $^{1}$ en un informe sobre las diez tendencias tecnológicas ${ }^{2}$ que regirán el panorama digital el próximo año, éstas alcanzarán un auge mundial -durante al menos la próxima década- en todos los ámbitos de la sociedad, afectando algunas de ellas al periodismo. De ahí la preocupación profesional y académica por estar adaptados a estas nuevas exigencias en el desempeño de la labor periodística.

El profesional de la Comunicación del siglo XXI no sólo se verá afectado por la irrupción de las nuevas tecnologías, sino que las nuevas necesidades del mercado ya han impuesto un perfil mucho más transversal, con mayor amplitud de miras, donde la comunicación informativa y la comunicación publicitaria y audiovisual son percibidas de modo unitario e indisociable. El periodista del futuro deberá redactar para diferentes medios, soportes y formatos, lo que le exigirá una mayor flexibilidad y formación complementaria en destrezas y habilidades tecnológicas.

\section{La necesaria formación en tecnologías para los futuros periodistas}

Si, como se ha puesto de manifiesto, las nuevas tecnologías están provocando un tsunami digital en la transformación de las organizaciones -incluyendo la empresa periodística-las Facultades de Comunicación deben dar soporte y respuesta a las necesidades formativas emergentes de este nuevo profesional del siglo XXI, en consonancia con las necesidades del mercado laboral. Las universidades deben acompañar e, incluso, liderar la formación en nuevas tecnologías, necesaria para atender a los vertiginosos avances tecnológicos y los requerimientos del sector productivo. El Espacio Europeo de Educación Superior (EEES) o Plan Bolonia (Comisión Europea, 1999) ha facilitado que las organizaciones empresariales puedan participar en el diseño de los planes de estudio. De este modo, empresa y universidad establecen un binomio perfecto para exista una retroalimentación adecuada. De nada serviría formar a profesionales en las universidades que no estuvieran formándose en las nuevas necesidades de comunicación que precisan las empresas a partir de la irrupción de estas nuevas tecnologías que afectan a los procesos de producción, edición, realización y publicación de noticias. Los periodistas, por tanto, deben aprovechar las ventajas que ofrecen las nuevas tecnologías en el desarrollo de la actualidad.

Casals (2006, pp. 65-66) considera una obligación incorporar las herramientas TIC en el proceso de enseñanza-aprendizaje del periodismo. Esta transición hacia el periodismo multimedia ha igualado en importancia al qué se comunica y cómo lo hace el periodista. Incluso en esta era de la imagen y de la inmediatez, podríamos asegurar que el cómo condicionará la forma de lo que comunicamos. Tejedor (2008, p. 25), en esta misma línea de pensamiento, es consciente de la revolución que supone Internet en el desempeño diario de las labores periodísticas y acentúa la necesidad de una formación competitiva de futuros profesionales de la comunicación online que les capacite para estar preparados y dar respuesta a los retos del nuevo escenario laboral. Verón y Sabes (2008, p. 113) señalan a las Facultades de Comunicación como agentes protagonistas para asumir el reto de formar en a los futuros comunicadores en materia tecnológica para capacitarles adecuadamente para trabajar en el mercado laboral. Además, ponen de manifiesto $(2008$, p. 107) la necesidad de hacer cambios sustanciales en los planes de estudio de Periodismo con la finalidad de que los estudiantes de estos Grados profundicen en los conceptos, fundamentos y técnicas del ciberperiodismo:

sí que tenemos que mostrar a nuestros universitarios qué es el ciberperiodismo, de eso no

tenemos ninguna duda, pero hay que cambiar otras muchas cosas dentro de los planes de

\footnotetext{
${ }^{1}$ Puede consultarse la información más completa en https://gtnr.it/2yjb2d3 (Consultado 14/12/2018).

${ }^{2}$ Inteligencia Artificial (AI), aplicaciones y analítica inteligente, intelligent things, digital twins, cloud to the edge, plataformas conversacionales, experiencias inmersivas, blockchain, event, riesgo adaptativo continuo y confianza.
} 
estudios ante una realidad tan cambiante en el mundo de la comunicación. El objetivo está en

lograr una formación adecuada y acorde con la situación actual, pero también pensando en su

futuro. (Verón y Sabes, p. 107)

Con esta visión cambiante del mundo de la comunicación también coincide Palomo Torres (2012, p. 681), quien reivindica la necesaria renovación constante de los estudios de Periodismo para adaptar sus contenidos a los contextos actuales.

Complementando la visión de estos investigadores, existen otros autores que han plasmado en sus trabajos la necesidad de incorporar la enseñanza del ciberperiodismo en los planes de estudio de los Grados de Periodismo y Comunicación (Martínez Albertos, 1997; Meso, 2008; Barranquero y Redondo, 2009; López, 2010; Orihuela, 2011; Cobo y Juárez, 2012; Acedo, Marta y Aparicci, 2013; Sánchez García y Campos, 2016). Todos ellos ponen de manifiesto la necesidad de formar en tecnologías a los futuros periodistas. Algunos de ellos , aun compartiendo la necesidad de formación en tecnologías, recalcan el hecho de no perder de vista los valores esenciales de la profesión: búsqueda de la verdad, verificación de fuentes, independencia informativa, control del poder, selección de noticias, análisis de los hechos, contraste de las fuentes, seguimiento de unos principios deontológicos, etc. El diseño de los nuevos planes de estudio tendrá como desafío, por tanto, la búsqueda del equilibrio entre la formación en tecnologías y la formación en los fundamentos que tradicionalmente han constituido la profesión periodística. La coyuntura educativa del EEES permite, en gran medida, esa flexibilidad de actualización y adaptación permanente (Sierra, 2010 ${ }^{\mathrm{a}}$, pp. 156157).

\section{Objeto, Hipótesis y Objetivos de la Investigación}

El objeto del presente trabajo de investigación es conocer cómo está implementada la formación en nuevas tecnologías en los Grados de Periodismo y Comunicación en las Universidades españolas. Hemos planteado las siguientes hipótesis de partida:

H1. Existe una baja carga crediticia en materia de tecnologías en los Grados de Periodismo/Comunicación españoles.

H2. Las tecnologías emergentes que más cabida tienen en los planes de estudio de Periodismo son la que tienen que ver con el tratamiento y procesamiento de grandes datos (periodismo de datos/big data).

H3. Los softwares tecnológicos para entrenar las competencias tecnológicas son fundamentalmente de pago (licencia comercial).

El objetivo general es realizar una radiografía de cómo han insertado las Facultades de Ciencias de la Comunicación en España la formación en Tecnología dentro de los nuevos diseños de los planes de estudio a partir de la entrada en vigor del Real Decreto 1393/2007, de 29 de octubre, por el que se establece la ordenación de las enseñanzas universitarias oficiales y la posterior actualización Real Decreto 861/2010, de 2 de julio. De este objetivo general se derivan una serie de objetivos específicos:

1. Descubrir qué tecnologías son las que abordan los alumnos del Grado de Periodismo/Comunicación en las Universidades españolas.

1.1. Indagar bajo qué modalidad (optativa/obligatoria) se presentan las asignaturas relacionadas con la tecnología dentro del plan de estudios. 
2. Analizar cuáles son los softwares empleados para comprender los fundamentos de las diferentes tecnologías estudiadas.

2.1. Descubrir cuáles son los programas informáticos que emplean para la edición de vídeo y postproducción audiovisual; edición de imágenes estáticas e ilustración; edición y postproducción sonora; creación de APP y medición de uso; 3D; creación de páginas web y analítica.

3. Estudiar cuáles son los contenidos digitales abordados durante la formación en el Grado de Periodismo/Comunicación.

4. Averiguar cuáles son las universidades que mayor número de asignaturas ofrecen (en los planes de estudio de Periodismo/Comunicación) relacionadas con las tecnologías.

\section{Metodología de investigación}

Este trabajo está basado en un enfoque de investigación cualitativa (Rodríguez, Gil y García, 1996), utilizando la técnica del análisis de documentos ${ }^{3}$. Los hallazgos potenciales de las guías docentes de las asignaturas de cada uno de los planes de estudio se localizaron siguiendo procedimientos de investigación del análisis de contenido (Berelson, 1952; Martín López, 1963; Krippendorff, 1990). A partir de esta metodología, se estableció un sistema categorial consistente en clasificar la información que contenían los documentos normativos a partir de un criterio previamente definido.

Tabla 1. Sistema categorial del análisis de las asignaturas de tecnologías de los Grados de Periodismo/Comunicación en España.

\begin{tabular}{|c|c|}
\hline Dimensión & Ítem de análisis \\
\hline $\begin{array}{l}\text { Diseño planes de estudio } \\
\text { Periodismo/Comunicación }\end{array}$ & $\begin{array}{l}\text { - Número de créditos destinados a la formación } \\
\text { en tecnología } \\
\text { - Carácter optativo y obligatorio de los mismos } \\
\text { - Número de asignaturas en la que se forma en } \\
\text { competencias tecnológicas }\end{array}$ \\
\hline Tecnología & $\begin{array}{l}\text { - Tecnologías estudiadas } \\
\text { - Contenidos digitales abordados }\end{array}$ \\
\hline $\begin{array}{l}\text { Softwares que dan soporte a la } \\
\text { formación tecnológica }\end{array}$ & $\begin{array}{ll}\text { - } & \text { Edición de Imágenes estáticas e ilustración } \\
\text { - } & \text { Edición de vídeo y postproducción } \\
\text { - } & \text { Edición de audio y postproducción sonora } \\
\text { - } & \text { Creación de apps y medición de uso } \\
\text { - } & \text { 3D } \\
\text { - } & \text { Creación web y posterior analítica } \\
\text { - } & \text { Estadística } \\
\end{array}$ \\
\hline
\end{tabular}

Fuente: elaboración propia.

Para elaborar el análisis, se han establecido los siguientes aspectos:

a) Período temporal: mes de noviembre de 2018.

b) Muestra: todas las Universidades (38) que contaran en su oferta académicas con la titulación de Periodismo/Comunicación ${ }^{4 \cdot}$ En total, se analizaron 38 Grados.

c) Acotación territorial: España.

\footnotetext{
${ }^{3}$ Se analizaron todas las guías docentes de las Universidades españolas que poseen Grados de Periodismo o Comunicación, con la excepción de la Universidad Europea de Madrid y de IE University, que no tienen publicadas en acceso abierto en la web las guías docentes.

${ }^{4}$ En este análisis hemos excluido a los Centros Adscritos Universitarios, puesto que la formación que era objeto de análisis la podíamos obtener de la Universidad de referencia de la que depende el Centro adscrito en cuestión.
} 
d) Unidades de análisis: tipo de tecnologías, tipo de contenidos digitales, softwares que emplean para afianzar el conocimiento en tecnología, así como el número de asignaturas y créditos que contemplan formación en nuevas tecnologías.

Una vez fijados estas variables, se realiza un vaciado de contenido de las guías docentes de 1912 asignaturas $^{5}$ de todos los planes de estudio de Periodismo/Comunicación.

Tabla 2. Grados y Universidades que han participado en el estudio.

\begin{tabular}{|c|c|c|c|c|c|}
\hline Universidad & Tipo & Denominación & Universidad & Tipo & Denominación \\
\hline $\begin{array}{l}\text { Universidad de } \\
\text { Navarra }\end{array}$ & Privada & Periodismo & Universidad de Vic & Pública & Periodismo \\
\hline $\begin{array}{l}\text { Universidad } \\
\text { Francisco de } \\
\text { Vitoria } \\
\end{array}$ & Privada & Periodismo & $\begin{array}{l}\text { Universidad de } \\
\text { Málaga }\end{array}$ & Pública & Periodismo \\
\hline $\begin{array}{l}\text { Universidad } \\
\text { Europea del } \\
\text { Atlántico }\end{array}$ & Privada & Periodismo & $\begin{array}{l}\text { Universidad de } \\
\text { Castilla- La Mancha }\end{array}$ & Pública & Periodismo \\
\hline $\begin{array}{l}\text { Universidad Camilo } \\
\text { José Cela }\end{array}$ & Privada & Comunicación & $\begin{array}{l}\text { Universidad Carlos } \\
\text { III de Madrid }\end{array}$ & Pública & Periodismo \\
\hline UDIMA & Privada & Periodismo & $\begin{array}{l}\text { Universitat } \\
\text { Autónoma } \\
\text { Barcelona }\end{array}$ & Pública & Periodismo \\
\hline $\begin{array}{l}\text { Universidad } \\
\text { Pontificia de } \\
\text { Salamanca }\end{array}$ & Privada & Periodismo & $\begin{array}{l}\text { Universidad Miguel } \\
\text { Hernández }\end{array}$ & Pública & Periodismo \\
\hline $\begin{array}{l}\text { Universidad de } \\
\text { Deusto }\end{array}$ & Privada & Comunicación & $\begin{array}{ll}\text { Universidade } & \text { de } \\
\text { Santiago } & \text { de } \\
\text { Compostela } & \\
\end{array}$ & Pública & Periodismo \\
\hline $\begin{array}{l}\text { Universitat Abat } \\
\text { Oliba CEU }\end{array}$ & Privada & Periodismo & Universitat Jaume I & Pública & Periodismo \\
\hline $\begin{array}{l}\text { Universidad San } \\
\text { Jorge }\end{array}$ & Privada & Periodismo & $\begin{array}{l}\text { Universitat Oberta } \\
\text { de Cataluña (UOC) }\end{array}$ & Pública & Comunicación \\
\hline $\begin{array}{l}\text { Universidad } \\
\text { Antonio de Nebrija }\end{array}$ & Privada & Periodismo & $\begin{array}{ll}\text { Universidad de } \\
\text { Sevilla }\end{array}$ & Pública & Periodismo \\
\hline UNIR & Privada & Comunicación & $\begin{array}{l}\text { Universidad del País } \\
\text { Vasco }\end{array}$ & Pública & Periodismo \\
\hline $\begin{array}{l}\text { Universidad } \\
\text { Europea Miguel de } \\
\text { Cervantes }\end{array}$ & Privada & Periodismo & $\begin{array}{l}\text { Universidad de La } \\
\text { Laguna }\end{array}$ & Pública & Periodismo \\
\hline $\begin{array}{l}\text { Universitat Pompeu } \\
\text { Fabra }\end{array}$ & Privada & Periodismo & $\begin{array}{l}\text { Universitat Rovira i } \\
\text { Virgili }\end{array}$ & Pública & Periodismo \\
\hline $\begin{array}{l}\text { Universitat Ramón } \\
\text { Llull }\end{array}$ & Privada & $\begin{array}{l}\text { Periodismo y } \\
\text { comunicación } \\
\text { corporativa }\end{array}$ & $\begin{array}{l}\text { Universidad } \\
\text { Valladolid }\end{array}$ & Pública & Periodismo \\
\hline $\begin{array}{l}\text { Universidad } \\
\text { Católica de Murcia }\end{array}$ & Privada & Periodismo & $\begin{array}{l}\text { Universidad } \\
\text { Complutense } \\
\text { Madrid }\end{array}$ & Pública & Periodismo \\
\hline $\begin{array}{l}\text { Universitat } \\
\text { Internacional } \\
\text { Cataluña }\end{array}$ & Privada & Periodismo & $\begin{array}{l}\text { Universidad } \\
\text { Juan Carlos }\end{array}$ & Pública & Periodismo \\
\hline $\begin{array}{l}\text { Universidad San } \\
\text { Pablo CEU }\end{array}$ & Privada & Periodismo & $\begin{array}{l}\text { Universidad } \\
\text { Murcia }\end{array}$ & Pública & Periodismo \\
\hline Universidad & Privada & Periodismo & Universidad & Pública & Periodismo \\
\hline
\end{tabular}

\footnotetext{
${ }^{5}$ Nota aclaratoria: en todos los análisis realizados en cada uno de los Grados se ha establecido que las materias de TFG y Prácticas externas no tienen relación con impartición de nuevas tecnologías.
} 
RLCS, Revista Latina de Comunicación Social, 75, 189-206

[Investigación] DOI: 10.4185/RLCS-2020-1422| ISSN 1138-5820 | Año 2020

\begin{tabular}{|l|l|l|l|l|l|}
\hline $\begin{array}{l}\text { Cardenal Herrera } \\
\text { CEU }\end{array}$ & & & Zaragoza & & \\
\hline $\begin{array}{l}\text { Universidad } \\
\text { Pontificia } \\
\text { Comillas de }\end{array}$ & Privada & $\begin{array}{l}\text { Comunicación } \\
\text { Internacional }\end{array}$ & $\begin{array}{l}\text { Universitat de } \\
\text { Valencia }\end{array}$ & Pública & Periodismo \\
\hline
\end{tabular}

Fuente: elaboración propia.

\section{Resultados de la investigación: descripción y análisis}

\subsection{Resultados referidos a la implantación en créditos dentro de los programas de los Grados de Periodismo/Comunicación}

De las 38 universidades y Grados analizados, se estudiaron los contenidos curriculares de 1912 guías docentes $^{6}$, de las cuales $370^{7}(19,3 \%)$ tienen relación con la formación en nuevas tecnologías de forma directa o indirecta.

Veamos los datos en el global y su conjunto para, posteriormente, establecer una comparativa entre Universidades públicas y privadas, así como del carácter/modalidad mediante los que se imparten los contenidos tecnológicos.

Tabla 3. Resultados globales de asignaturas relacionadas con las tecnologías que se estudian en los Grados de Periodismo/Comunicación en las Universidades españolas.

\begin{tabular}{|l|r|r|r|r|l|}
\hline \multicolumn{1}{|c|}{ Universidad } & $\begin{array}{c}\text { Número de } \\
\text { asignaturas }\end{array}$ & $\begin{array}{c}\text { Peso en } \\
\text { Créditos }\end{array}$ & $\begin{array}{c}\text { OB } \\
\text { (obligatoria) }\end{array}$ & $\begin{array}{c}\text { OP } \\
\text { (optativa) }\end{array}$ & \multicolumn{1}{|c|}{ Tipo } \\
\hline Universidad de Navarra & 23 & 123 & 39 & 84 & Privada \\
\hline Universidad de Vic & 14 & 60 & 54 & 6 & Pública \\
\hline Universidad de Málaga & 14 & 84 & 48 & 36 & Pública \\
\hline $\begin{array}{l}\text { Universidad Castilla-La } \\
\text { Mancha }\end{array}$ & 14 & 84 & 30 & Pública \\
\hline Universidad Francisco de Vitoria & 13 & 60 & 45 & 15 & Privada \\
\hline Universidad Carlos III de Madrid & 13 & 75 & 57 & 18 & Pública \\
\hline $\begin{array}{l}\text { Universitat Autónoma } \\
\text { Barcelona }\end{array}$ & 12 & 72 & 72 & 0 & Pública \\
\hline Universidad Camilo José Cela & 12 & 72 & 48 & 24 & Privada \\
\hline UDIMA Pontificia & 11 & 66 & 54 & 12 & Privada \\
\hline $\begin{array}{l}\text { Universidad } \\
\text { Salamanca }\end{array}$ & 11 & 66 & 66 & 0 & Privada \\
\hline Universidad de Deusto & 11 & 66 & 66 & 0 & Privada \\
\hline Universidad Miguel Hernández & 11 & 66 & 66 & 0 & Pública \\
\hline Universitat Abat Oliba CEU & 11 & 63 & 63 & 48 & Privada \\
\hline Universidad San Jorge & 11 & 60 & 60 & Privada \\
\hline Universidad Antonio de Nebrija & & 66 & Privada \\
\hline
\end{tabular}

\footnotetext{
${ }^{6}$ Llama poderosamente la atención cómo existen asignaturas en las que, con denominaciones apartemente ajenas a la tecnología, el profesor ha decido dar a ese contenido una visión tecnológica del tema y, sin embargo, en otras materias de las que, por su propia denominación, se podría intuir relación directa con la tecnología, el profesor ha abordado los contenidos desde ópticas más teóricas y alejadas de los fundamentos tecnológicos a priori requeridos

${ }^{7}$ Es posible que esa cifra pueda incrementarse en el curso 19/20 puesto, que la Universidad Europea del Atlántico se encuentra actualmente en el tercer año de implantación del Grado de Periodismo.
} 
RLCS, Revista Latina de Comunicación Social, 75, 189-206

[Investigación] DOI: 10.4185/RLCS-2020-1422| ISSN 1138-5820 | Año 2020

\begin{tabular}{|l|r|r|r|r|l|} 
Universidade de Santiago de & 10 & 60 & 60 & 0 & Pública \\
\hline UNIR & 10 & 40 & 36 & 4 & Privada \\
\hline $\begin{array}{l}\text { Universidad Europea Miguel de } \\
\text { Cervantes }\end{array}$ & 9 & 54 & 42 & 12 & Privada \\
\hline Universitat Jaume I & 9 & 54 & 48 & 6 & Pública \\
\hline Universitat Pompeu Fabra & 9 & 44 & 8 & 36 & Privada \\
\hline $\begin{array}{l}\text { Universitat Oberta de Cataluña } \\
\text { (UOC) }\end{array}$ & 9 & 54 & 36 & 18 & Pública \\
\hline Universidad de Sevilla & 9 & 54 & 36 & 18 & Pública \\
\hline Universidad del País Vasco & 9 & 54 & 54 & 0 & Pública \\
\hline Universitat Ramón Llull & 9 & 32 & 29 & 3 & Privada \\
\hline Universidad de La Laguna & 8 & 48 & 36 & 12 & Pública \\
\hline Universitat Rovira i Virgili & 8 & 57 & 39 & 18 & Pública \\
\hline Universidad de Valladolid & 8 & 48 & 36 & 12 & Pública \\
\hline $\begin{array}{l}\text { Universidad Complutense de } \\
\text { Madrid }\end{array}$ & 8 & 48 & 30 & 18 & Pública \\
\hline Universidad Rey Juan Carlos & 8 & 48 & 42 & 6 & Pública \\
\hline Universidad de Murcia & 8 & 54 & 36 & 18 & Pública \\
\hline Universidad Católica de Murcia & 8 & 48 & 48 & 0 & Privada \\
\hline $\begin{array}{l}\text { Universitat Internacional de } \\
\text { Cataluña }\end{array}$ & 7 & 29 & 26 & 3 & Privada \\
\hline Universidad de Zaragoza & 6 & 39 & 27 & 12 & Pública \\
\hline Universitat de Valencia & 6 & 33 & 24 & 9 & Pública \\
\hline Universidad San Pablo CEU & 36 & 36 & 0 & Privada \\
\hline $\begin{array}{l}\text { Universidad Cardenal Herrera } \\
\text { CEU }\end{array}$ & 6 & 36 & 0 & Privada \\
\hline Universidad Europea del Atlántico & $\mathbf{3 7 0 4}$ ECTS & $\mathbf{1 6 5 6}$ ECTS & $\mathbf{4 4 8}$ ECTS & Privada \\
\hline Universidad Pontificia de Comillas & & & & \\
\hline TOTAL & & 36 & & & \\
\hline
\end{tabular}

Fuente: elaboración propia.

De los 2104 ECTS en los que se imparten contenidos relacionados con las nuevas tecnologías, el $78,7 \%$ de los créditos se imparten como materias obligatorias, mientras que el $22 \%$ restante corresponde a créditos de carácter optativo, en función de la especialización o itinerario que decide escoger el alumno. Si se hiciera un promedio del número de asignaturas relacionadas con las nuevas tecnologías que se imparten en los Grados de Periodismo/Comunicación en España, se situaría alrededor de 10 asignaturas. A la vista de los resultados obtenidos, podemos confirmar H1, puesto que parece insuficiente y escasa la oferta de asignaturas de carácter tecnológico dentro de los planes de estudio de Periodismo/Comunicación.

Veamos en la siguiente tabla una comparativa entre la formación en tecnologías en los Grados de Periodismo/Comunicación en función de la titularidad de la Universidad. 
Tabla 4. Comparativa en formación en Tecnología en los Grados de Periodismo/Comunicación en función de la titularidad.

\begin{tabular}{|c|c|c|c|c|}
\hline Tipo de Universidad & $\begin{array}{c}\mathrm{N}^{\mathrm{o}} \text { de } \\
\text { asignaturas }\end{array}$ & Peso en créditos & Obligatorias & Optativas \\
\hline Universidades Públicas & 184 & 1092 & 855 & 237 \\
\hline Universidades Privadas & 186 & 1012 & 801 & 211 \\
\hline TOTAL & 370 & 2104 ECTS & 1656 ECTS & 448 ECTS \\
\hline
\end{tabular}

Fuente: elaboración propia.

En ambos casos se observa una gran igualdad en cuanto al número de asignaturas, incluso en el número de créditos que representan, así como la proporcionalidad en el número de créditos en obligatorias y optativas, teniendo en cuenta que a la Universidad Europea del Atlántico, de titularidad privada, le falta por implantar el cuarto año del Grado. Podemos, por tanto, afirmar que apenas existen diferencias significativas en el cómputo global a la hora de establecer la estrategia de incorporar formación en nuevas tecnologías. Las cinco universidades que ofrecen un mayor número de asignaturas relacionadas con las nuevas tecnologías serían, por orden de prelación, Universidad de Navarra (23), Universidad de Vic (14), Universidad de Málaga (14), Universidad de Castilla-La Mancha (14) y la Universidad Francisco de Vitoria (13) y Universidad Carlos III de Madrid (13), que ocupan conjuntamente la quinta posición. Sin embargo, si estableciéramos ese ranking por el peso en créditos, las posiciones anteriores variarían y el resultado sería: Universidad de Navarra (120 ECTS), Universidad de Málaga (84 ECTS), Universidad de Castilla-La Mancha (84 ECTS), Universidad Carlos III (75 ECTS) y la Universitat Autónoma de Barcelona, igualada con la Universidad Camilo José Cela (72 ECTS).

\subsection{Resultados referidos a las tecnologías emergentes abordadas en los planes de estudio y softwares empleados para adquirir y entrenar las competencias tecnológicas}

Veamos a continuación cuáles han sido las tecnologías emergentes que más se han tratado en las asignaturas a partir del análisis de la información publicada en el apartado de contenidos de las guías docentes.

Tabla 5. Tecnologías emergentes abordadas en los Grados de Periodismo/Comunicación en las Universidades españolas.

\begin{tabular}{|l|r|r|}
\hline Tecnologías emergentes abordadas & $\begin{array}{c}\mathrm{N}^{\mathrm{o}} \text { de } \\
\text { asignaturas }\end{array}$ & $\begin{array}{c}\text { Porcentaje sobre } \\
\text { las 370 asignaturas } \\
\text { de tecnología }\end{array}$ \\
\hline Big data y Periodismo de datos & 25 & $6,75 \%$ \\
\hline Fotografía y Vídeo $360^{\circ}$ & 6 & $1,62 \%$ \\
\hline Cloud computing & 5 & $1,35 \%$ \\
\hline Realidad Aumentada & 4 & $1,08 \%$ \\
\hline Realidad Virtual & 4 & $1,08 \%$ \\
\hline Internet de las cosas & 3 & $0,81 \%$ \\
\hline Inteligencia artificial & 3 & $0,81 \%$ \\
\hline Televisión Conectada & 2 & $0,54 \%$ \\
\hline
\end{tabular}

Fuente: elaboración propia. 
En la tabla V se puede observar cómo los porcentajes son realmente bajos. Muy lentamente, los Grados de Periodismo/Comunicación van incorporando las tecnologías emergentes que son tendencia para los próximos años y que están copando varios sectores de la sociedad actual; y, como no podía ser de otro modo, también aplican al sector de la información. En este sentido, podemos destacar cómo el Periodismo de datos (big data) es actualmente el que ha entrado con más fuerza. En esta ocasión, de nuevo se confirma $\mathrm{H} 2$, puesto que es el big data, dentro de las tecnologías emergentes, la que más penetración tiene en los planes de estudio a la vista de los datos reflejados.

A continuación, se analizan los softwares empleados para comprender los fundamentos de las diferentes tecnologías estudiadas (emergentes) y audiovisuales más tradicionales.

Tabla 6. Softwares empleados para el aprendizaje de la edición y postproducción de vídeo.

\begin{tabular}{|l|r|}
\hline $\begin{array}{c}\text { Softwares relacionados con la edición } \\
\text { de vídeo y la postproducción }\end{array}$ & $\begin{array}{c}\text { Número de } \\
\text { asignaturas que } \\
\text { emplean el } \\
\text { software }\end{array}$ \\
\hline Avid & 31 \\
\hline Adobe Premiere & 24 \\
\hline Adobe After Effects & 10 \\
\hline Final Cut & 5 \\
\hline Cinema 4D & 2 \\
\hline Sony Vegas & 1 \\
\hline Pinacle Studio & 1 \\
\hline Cine Larra & 1 \\
\hline Trakax & 1 \\
\hline Mistika & 1 \\
\hline HyperEngine-AV & 1 \\
\hline
\end{tabular}

Fuente: elaboración propia.

Podemos apreciar cómo los softwares de referencia para el aprendizaje de la edición y postproducción de vídeo son AVID y las soluciones de Adobe: Premiere, fundamentalmente para la edición de vídeo, y After Effects, para la postproducción. Ambos softwares son de pago, pero ofrecen licencias educacionales. Tanto Avid como Adobe son estándares en la industria del audiovisual para esos cometidos. También podemos apreciar cómo las Universidades optan por ofrecer a los alumnos software libre o de código abierto para esos cometidos, como son los programas informáticos de Cinelerra ${ }^{8}, \operatorname{Trakax}^{9}$ o HyperEngine-AV ${ }^{10}$.

Veamos en la siguiente tabla cuáles son los programas que emplean para aprender a editar e ilustrar imágenes.

\footnotetext{
${ }^{8}$ Es un programa libre para la edición de vídeo bajo el sistema operativo GNU/Linux.

${ }^{9}$ TrakAx PC edita audio y vídeo para realizar mejoras de sonido, cortes y montajes.

${ }^{10}$ Software de autoría multimedia para capturar, editar, organizar, procesar y exportar vídeo, audio, texto y crear películas con calidad de DVD y presentaciones de diapositivas.
} 
RLCS, Revista Latina de Comunicación Social, 75, 189-206

[Investigación] DOI: 10.4185/RLCS-2020-1422| ISSN 1138-5820| Año 2020

Tabla 7. Softwares relacionados con la edición de imágenes estáticas e ilustración.

\begin{tabular}{|l|r|}
\hline $\begin{array}{c}\text { Softwares relacionados con la } \\
\text { edición de imágenes estáticas } \\
\text { e ilustración }\end{array}$ & $\begin{array}{c}\text { Número de } \\
\text { asignaturas que } \\
\text { emplean ese software }\end{array}$ \\
\hline Adobe Photoshop & 66 \\
\hline Adobe Indesign & 58 \\
\hline Adobe Ilustrator & 31 \\
\hline Adobe Flash & 6 \\
\hline QuarkXPress & 4 \\
\hline GIMP & 3 \\
\hline Inkscape & 3 \\
\hline Adobe Bridge & 2 \\
\hline Camera RAW & 1 \\
\hline Adobe Lightroom & 1 \\
\hline Adobe Experience Design & 1 \\
\hline
\end{tabular}

Fuente: elaboración propia.

En este campo, las soluciones de Adobe (Photoshop, Indesign, Ilustrator, Lightroom Experience Design y Camera Raw) son las más extendidas en el mercado profesional de la Comunicación periodística, publicitaria y audiovisual y la Universidad es fiel reflejo de ello. QuarkXpress, que fue muy empleado en la década de los noventa, hoy en día se encuentra obsoleto con respecto a lo que los softwares de la competencia pueden hacer. Por otro lado, en la tabla aparecen dos soluciones de código abierto y que son free: Inkscape ${ }^{11} \mathrm{y} \mathrm{GIMP}^{12}$.

En lo referente a la edición y postproducción de audio, se observa que el software más extendido en el aprendizaje es Audacity, que es una solución gratuita de software libre; seguido por los tres softwares de referencia del mercado profesional. Aquí se aprecia que son escasas las asignaturas que hacen referencia al tratamiento de captación, edición, reproducción y postproducción sonora. Sería interesante que los responsables del diseño de planes de estudios de Periodismo/Comunicación valorasen incorporar más materias que refuercen el lenguaje y la técnica del sonido, porque son capitales en la creación de mensajes.

Tabla 8. Softwares relacionados con el audio, la creación de app y 3D.

\begin{tabular}{|l|r|}
\hline $\begin{array}{c}\text { Softwares relacionados con la edición de } \\
\text { audio y la postproducción sonora }\end{array}$ & $\begin{array}{c}\text { Número de asignaturas } \\
\text { que emplean ese software }\end{array}$ \\
\hline Audacity & 12 \\
\hline Adobe Audition & 2 \\
\hline Pro Tools & 2 \\
\hline Logic Audio & 1 \\
\hline
\end{tabular}

\footnotetext{
${ }^{11}$ Inkscape es un editor de gráficos vectoriales libre y de código abierto. Inkscape puede crear y editar diagramas, líneas, gráficos, logotipos, e ilustraciones complejas.

${ }_{12}$ GIMP es un programa de edición de imágenes digitales en forma de mapa de bits, tanto dibujos como fotografías. Es un programa libre y gratuito. Forma parte del proyecto GNU y está disponible bajo la Licencia Pública General de GNU o GNU Lesser General Public License.
} 


\begin{tabular}{|l|r|}
\hline $\begin{array}{l}\text { Softwares relacionados con la creación de } \\
\text { app y medición de uso }\end{array}$ & $\begin{array}{l}\text { Número de asignaturas } \\
\text { que emplean ese software }\end{array}$ \\
\hline App Annie & 1 \\
\hline App Inventor & 1 \\
\hline Google App Maker & 1 \\
\hline Softwares relacionados con 3D & Número de asignaturas \\
\hline Sketch up & 2 \\
\hline 3ds Studio Max & 1 \\
\hline Visual SFM & 1 \\
\hline
\end{tabular}

Fuente: elaboración propia.

En lo que respecta a la creación de app, sólo algunas Uuniversidades, como Navarra con la asignatura Entertainment Apps Development y la Camilo José Cela con la materia Apps and Communication, han considerado este nicho como una parte necesaria a explorar como vía de futura empleabilidad de sus egresados.

Se puede comprobar, de este modo, cómo de forma natural el Periodismo se apoya en materias originalmente propias de la Comunicación Audiovisual para afianzar sus conocimientos tecnológicos en pro de un profesional de la Comunicación más versátil y multimedia. Para abordar la tridimensionalidad de la realidad, existe una escasa introducción de esta formación en los planes de Periodismo. Tan sólo cuatro asignaturas de las 370 analizadas consideran la relevancia de la tecnología 3D en la formación en Periodismo del siglo XXI.

Tabla 9. Softwares relacionados con la estadística.

\begin{tabular}{|l|r|r|}
\hline $\begin{array}{c}\text { Softwares relacionados } \\
\text { con la estadística }\end{array}$ & $\begin{array}{c}\mathrm{N}^{\mathrm{o}} \text { de asignaturas } \\
\text { que lo utilizan }\end{array}$ & $\begin{array}{c}\text { Porcentaje sobre las 370 } \\
\text { asignaturas de tecnología }\end{array}$ \\
\hline SPSS & 23 & $6,21 \%$ \\
\hline Excel & 11 & $2,97 \%$ \\
\hline $\mathrm{R}$ & 2 & $0,54 \%$ \\
\hline
\end{tabular}

Fuente: elaboración propia.

Ya se apuntó en la introducción que el big data, a través del Periodismo de datos, era crucial para la interpretación que el periodista puede y debe hacer de determinados datos que los gobiernos e instituciones ponen a disposición de los usuarios a través del open data. El conocimiento de la estadística es fundamental para comprender mejor la profundidad del big data y su aplicación a través del periodismo de datos; tanto la extracción de los datos como la visualización de los mismos. Se aprecia que este tipo de software también es empleado en la asignatura de Métodos y Técnicas de Investigación en Comunicación, que supone la antesala a la materia de TFG.

En el apartado introductorio de este trabajo también se ponía de manifiesto la incidencia de determinados investigadores sobre la necesidad de actualizar los planes de estudio de Periodismo y adaptarlos a los requerimientos y necesidades del ciberperiodismo. De ahí que se pueda observar en la tabla siguiente los esfuerzos que las diferentes Universidades han realizado por incorporar en los planes de estudio materias relacionadas con la creación de contenido en Internet. 
RLCS, Revista Latina de Comunicación Social, 75, 189-206

[Investigación] DOI: 10.4185/RLCS-2020-1422| ISSN 1138-5820| Año 2020

Tabla 10. Softwares relacionados con la creación de contenido y analítica web.

\begin{tabular}{|c|c|c|}
\hline Tipología & $\begin{array}{l}\text { Software relacionado con la } \\
\text { creación y analítica web }\end{array}$ & $\begin{array}{c}\mathrm{N}^{\mathrm{o}} \text { de asignaturas que } \\
\text { emplean ese } \\
\text { software/lenguaje }\end{array}$ \\
\hline \multirow{7}{*}{ Gestor de Contenidos } & CMS (Wordpress) & 31 \\
\hline & CMS (WIX) & 3 \\
\hline & CMS (Joomla) & 3 \\
\hline & CMS (Drupal) & 2 \\
\hline & CMS (Bluegriffon) & 2 \\
\hline & Adobe Dreamweaver & 3 \\
\hline & Omeka $^{13}$ & 1 \\
\hline \multirow{6}{*}{ Programación } & HTML & 18 \\
\hline & CSS & 5 \\
\hline & XML & 3 \\
\hline & Javascript & 2 \\
\hline & $\mathrm{C}++$ & 2 \\
\hline & Processing & 1 \\
\hline Base de datos & MySQL & 2 \\
\hline \multirow{5}{*}{ Analítica web } & Google Analytics & 14 \\
\hline & Google Adwords & 2 \\
\hline & Google Rank & 1 \\
\hline & Google Trends & 1 \\
\hline & Omniture & 1 \\
\hline Herramientas webmaster & Google Search Console & 1 \\
\hline Transferencia de archivos & FTP & 3 \\
\hline
\end{tabular}

Fuente: elaboración propia.

A partir de la visualización de la tabla $\mathrm{X}$, se pueden extraer varias conclusiones:

a) Existe un mayor número de asignaturas que ponen especial énfasis en el aprendizaje de creación de contenidos en la web.

b) Dentro de los CMS (Content Management System) el más extendido a nivel formativo es WordPress.

c) Dentro de los lenguajes de programación, se trabaja bajo un entorno HTML (HyperText Markup Language).

d) Por último, para todo lo referente a la analítica web, las soluciones gratuitas de Google se posicionan como las más extendidas, como no podía ser de otra manera, ya que, según los datos de Statcounter de diciembre de 2018, Google es el buscador es el líder con un 92,9\% de cuota de mercado mundial.

\footnotetext{
${ }^{13}$ Omeka es un software libre, flexible y de código abierto pensado para la publicación en la web de colecciones digitales de bibliotecas, archivos, museos o cualquier otra institución que desee difundir su patrimonio cultural.
} 


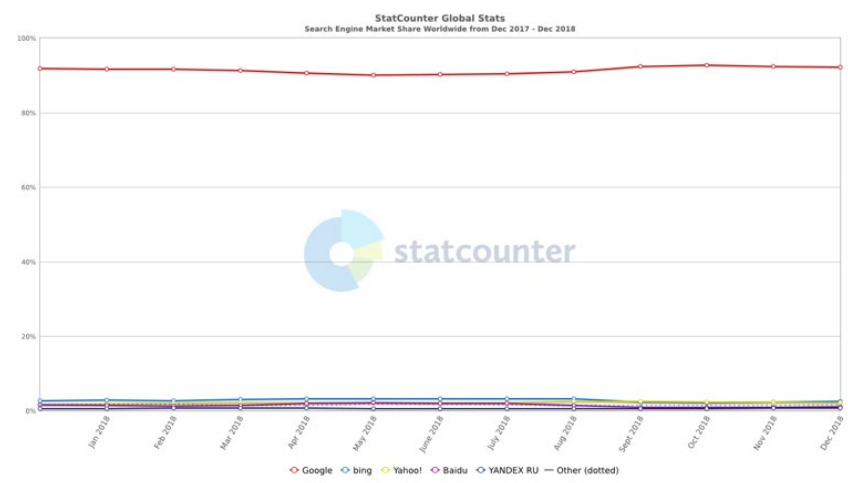

Figura 1: mercado de motores de búsqueda en todo el mundo ${ }^{14}$.

Fuente: Statcounter (2018).

\subsection{Resultados referidos a los contenidos digitales abordados en los planes de estudio de Periodismo/Comunicación}

Para finalizar este último apartado de resultados y atendiendo a uno de los objetivos planteados en el inicio, se muestran a continuación los resultados obtenidos acerca de cuáles son los contenidos digitales más abordados en los planes de estudio.

Tabla 11. Contenidos digitales que son abordados en las asignaturas de corte tecnológico en los planes de estudio de Periodismo/Comunicación.

\begin{tabular}{|c|c|c|c|}
\hline Contenidos digitales abordados & Tipos & $\begin{array}{c}\mathrm{N}^{\mathrm{o}} \mathrm{de} \\
\text { asignaturas }\end{array}$ & $\begin{array}{c}\text { Porcentaje } \\
\text { sobre las } 370 \\
\text { asignaturas de } \\
\text { tecnología }\end{array}$ \\
\hline Videojuegos & & 9 & $2,43 \%$ \\
\hline \multirow{13}{*}{ Redes Sociales } & Total redes & 42 & \multirow{13}{*}{$11,35 \%$} \\
\hline & Youtube & 7 & \\
\hline & LinkedIn & 4 & \\
\hline & Instagram & 4 & \\
\hline & Twitter & 6 & \\
\hline & Storify & 1 & \\
\hline & Google + & 1 & \\
\hline & Vimeo & 1 & \\
\hline & Snapchat & 1 & \\
\hline & WhatsApp & 1 & \\
\hline & Telegram & 1 & \\
\hline & Flickr & 1 & \\
\hline & Socialcam & 1 & \\
\hline App & & 6 & $1,62 \%$ \\
\hline Televisión digital (TDT) & & 27 & $7,29 \%$ \\
\hline Radio Digital & & 40 & $10,81 \%$ \\
\hline Periodismo digital & & 24 & $6,48 \%$ \\
\hline
\end{tabular}

Fuente: elaboración propia

\footnotetext{
${ }^{14}$ Obtenido de http://gs.statcounter.com/search-engine-market-share (Consultado 10/12/2018).
} 
Las redes sociales son, por tanto, objeto de especial importancia en el diseño de planes de estudio. Se aprecia cómo lo más abordado son las redes sociales $(11,35 \%)$, seguidas por la radio digital $(10,81 \%)$, la TDT $(7,29 \%)$ y el Periodismo digital $(6,48 \%)$.

\section{Conclusiones}

A partir de los datos obtenidos, es posible extraer las algunas conclusiones sobre cómo se van implementando los contenidos relacionados con la tecnología en los Grados de Periodismo/Comunicación en las Universidades españolas:

1. Las asignaturas relacionadas con las tecnologías se presentan fundamentalmente bajo la modalidad/carácter de obligatorias dentro del Plan de estudios.

2. Las Universidades públicas y privadas que albergan estudios de Periodismo han optado por una estrategia muy similar a la hora de introducir materias de corte tecnológico en los planes de estudio. La media global en España se sitúa alrededor de 10 materias tecnológicas en los planes de estudio.

3. Los programas de software privativo son fundamentalmente los que complementan la formación tecnológica del futuro periodista. En este sentido, destacan las siguientes licencias comerciales para entrenar cada una de las destrezas tecnológicas: AVID y el paquete Adobe para la edición y postproducción de imágenes fijas y en movimiento. Para el estudio de la tridimensionalidad, SkecthUp y Autodesk 3ds Studio Max son los estandarizados en la formación y en el mercado laboral. La solución aportada por IBM (SPSS) es la más extendida en la formación universitaria para comprender el tratamiento estadístico de los datos. Tan sólo Audacity, que es una aplicación para la edición y postproducción de audio, es la solución de software libre más extendida en la formación periodística. Estos datos permiten confirmar positivamente la hipótesis $\mathrm{H} 3$.

4. Los contenidos digitales que captan el foco de formación y atención dentro de los diseños de los planes de estudio son fundamentalmente las redes sociales, ya que se han convertido en un medio fundamental de propagación de la información, así como de consumo y distribución de productos informativos.

\section{Referencias bibliográficas}

Osuna Acedo, S., Marta Lazo, C. y Aparici Marino, R. A. (2013). Valores De La Formación Universitaria De Los Comunicadores En La Sociedad Digital. Más Allá Del Aprendizaje Tecnológico, Hacia Un Modelo Educomunicativo. Razón y Palabra, 81, 608-638. https://bit.ly/2tpI8HA

Barranquero, A., y Redondo, F. (2009). Análisis Comparado de los planes de Estudio de Periodismo antes y después de Bolonia. En VV.AA. (Coord.), Actas del I Congreso Internacional Latina de Comunicación Social (pp. 1-16). S. C. de Tenerife: Sociedad Latina de Comunicación Social. https://bit.ly/3b7RQzy

Berelson, B. (1952). Content analysis in communication research. Hafner.

Casals Carro, M. J. (2006). La enseñanza del periodismo y las nuevas tecnologías de la información y de la comunicación. Estudios sobre el mensaje periodístico, 12, 59-70. https://bit.ly/2u6yhXJ

Cobo, S., y Juárez, S. C. (2012). Internet para periodistas: kit de supervivencia para la era digital. UOC. 
Comisión Europea (1999). Declaración de Bolonia. https://bit.ly/36W6W7P

Fondevila Gascón, J. F. (2010). El ‘cloud journalism'. Un nuevo concepto de producción para el periodismo del siglo XXI. Observatorio (OBS*), 4(1), 19-35.

https://doi.org/10.15847/obsOBS412010315

Krippendorff, K. (1990). Metodología del análisis de contenido. Teoría y Práctica. Paidós.

Jiménez-Gómez, I., \& Mañas-Viniegra, L. (2018). Ofertas de empleo mejor remuneradas en comunicación y diseño: nuevos perfiles y efecto full-stack. Doxa Comunicación, 27, 239-251. https://doi.org/10.31921/doxacom.n27a12

Lerma Noriega, C. A. (2018). Retos para la educación del futuro periodista. Estudios sobre el Mensaje Periodístico, 24(2), 1433-1444. https://doi.org/10.5209/ESMP.62226

López García, S. (2010). La formación de los periodistas en el siglo XXI en Brasil, España, Portugal y Puerto Rico. Revista Latina de Comunicación Social, 65, 231-243. https://doi.org/10.4185/RLCS-65-2010-896-231-243

Marinho, S. (2012). Formação em jornalismo numa sociedade em mudança: modelos, percepções e práticas na análise do caso portugués (Tesis Doctoral). Braga: Universidade do Minho-Instituto de Ciências Sociais.

Martín López, E. (1963). El análisis de contenido. Revista de Estudios Politicos, 132, 45-64. https://bit.ly/2Sa3PUs

Martínez Albertos, J. L. (1997). El ocaso del periodismo. CIMS.

Meso Ayerdi, K. (2003). La formación del periodista digital. Chasqui. Revista Latinoamericana de Comunicación, 84, 5-11. https://doi.org/10.16921/chasqui.v0i84.1497

Orihuela, J. L. (2011). 80 claves sobre el futuro del periodismo. Anaya.

Palomo Torres, M. B. (2012). Los efectos de la enseñanza global del periodismo. Análisis de la influencia anglosajona en el aprendizaje de las nuevas tecnologías informativas. Estudios sobre el mensaje periodístico, 18, 681-689. https://doi.org/10.5209/rev_ESMP.2012.v18.40947

Real Decreto 1393/2007, de 29 de octubre, por el que se establece la ordenación de las enseñanzas universitarias oficiales. Boletín Oficial del Estado, 260, de 30 de octubre de 2007, 44037.

Real Decreto 861/2010, de 2 de julio, por el que se modifica el Real Decreto 1393/2007, de 29 de octubre, por el que se establece la ordenación de las enseñanzas universitarias oficiales. Boletín Oficial del Estado, 161, de 3 de julio de 2010, 58454.

Rodríguez, G., Gil, J., y García, E. (1996). Metodología de la investigación cualitativa. Aljibe.

Rosique Cedillo, G. (2013). Los estudios universitarios de periodismo en España. La adaptación al EEES y la formación de periodistas en competencias digitales. RIESED: Revista Internacional de Estudios sobre Sistemas Educativos, 1(1-2), 117-132. https://bit.ly/2vPuTB3 
Salaverría, R., y García Avilés, J. A. (2008). La convergencia tecnológica en los medios de comunicación: retos para el periodismo. Trípodos, 23, 31-47. https://bit.ly/36QrYVv

Sánchez-García, P., y Campos-Domínguez, E. (2016). La formación de los periodistas en nuevas tecnologías antes y después del EEES. El caso español. Trípodos, 38, 161-179. https://bit.ly/2RRLH2F

Sánchez-García, P., y Marinho, S. (2016). La formación digital en los programas de periodismo de España y Portugal. Andamios, 13(31), 339-361. https://doi.org/10.29092/uacm.v13i31.439

Scolari, C. A., Micó Sanz, J. L., Navarro Güere, H., y Pardo, H. (2008). El periodista polivalente. Transformaciones en el perfil del periodista a partir de la digitalización de los medios audiovisuales catalanes. Zer. Revista de Estudios de Comunicación, 13(25), 37-60. https://bit.ly/2RSs9vc

Sierra Sánchez, J. (2010a). Competencias profesionales y empleo en el futuro periodista. El caso de los estudiantes de periodismo de la Universidad San Pablo CEU.Icono14, 8(2), 156-175. https://doi.org/10.7195/ri14.v8i2.274

Sierra Sánchez, J. (Coord.) (2010b). Los estudios de Ciencias de la Comunicación en el EEES. Fragua.

Sierra Sánchez, J., y Cabezuelo Lorenzo, F. (2009). La formación en competencias del futuro periodista digital en Aragón. El caso de la Universidad San Jorge. En F. Sabés Turmo, \& J. J. Verón Lassa (Coord.), Retos del Periodismo Digital. Reflexiones desde la Universidad (pp. 223238). Asociación de la Prensa de Aragón.

Tejedor, S. (2008). Ciberperiodismo y universidad: diagnósticos y retos de la enseñanza del periodismo on-line. Anàlisi: quaderns de comunicació i cultura, 36, 25-39. https://bit.ly/2OjwmWz

Verón Lassa, J. J., y Sabés Turmo, F. (2008). Las nuevas tecnologías en la formación del periodista. Una necesidad real e inmediata. Anàlisi: quaderns de comunicació $i$ cultura, 36, 105-114. https://bit.ly/36RWezi

\section{AUTORES:}

\section{Javier Sierra Sánchez}

Departamento de Ciencias de la Comunicación Aplicada. Universidad Complutense de Madrid. España.

Doctor en Ciencias de la Información por la Universidad Complutense de Madrid (UCM) y licenciado en Comunicación Audiovisual y Periodismo por la misma universidad. Master oficial en Marketing y Comunicación Corporativa por la Universidad San Jorge. Experto en RRPP Internacionales por la UCM. Experto en Protocolo y Ceremonial del Estado e Internacional por la Universidad de Oviedo y la Escuela Diplomática de Madrid. Actualmente ejerce docencia como profesor contratado doctor en la Universidad Complutense de Madrid.

javiersierrasanchez@pdi.ucm.es

Índice $\mathbf{H}: 12$

Orcid ID: https://orcid.org/0000-0001-8572-7564

Google Scholar: https://scholar.google.es/citations?user=oGqa6PgAAAAJ\&hl=es 


\section{Sheila Liberal Ormaechea}

Facultad de Comunicación. Universidad Francisco de Vitoria. España.

Doctora en Ciencias de la Información por la Universidad Complutense de Madrid (2012); posee un Máster Universitario en Marketing y Comunicación Corporativa por la Universidad San Jorge de Zaragoza (2008), posee un Máster en Dirección Comercial y Maketing de ESIC (2003). Es Licenciada en Publicidad y Relaciones Públicas por la Universidad de Navarra (2001). Actualmente ejerce como Vicedecana de Investigación en la Facultad de Comunicación de la Universidad Francisco de Vitoria.

s.liberal.prof@ufv.es

Índice H: 3

Orcid ID: https://orcid.org/0000-0003-0449-3204

Google Scholar: https://scholar.google.es/citations?hl=en\&user=XXd6sYEAAAAJ

\section{Luis Mañas-Viniegra}

Departamento de Ciencias de la Comunicación Aplicada. Universidad Complutense de Madrid.

España.

Profesor Ayudante Doctor en el Departamento de Ciencias de la Comunicación Aplicada de la Universidad Complutense de Madrid. Doctor en Comunicación Audiovisual y Publicidad, es Licenciado en Periodismo y en Publicidad y Relaciones Públicas. Ha sido profesor en la Universidad Carlos III de Madrid, Universidad Rey Juan Carlos y Universidad de Valladolid. Es miembro del grupo de investigación Complutense de Gestión de Marca y Comunicación Integrada e IP del proyecto de innovación "Mapa visual de orientación profesional para el Grado en Publicidad y Relaciones Públicas".

lmanas@ucm.es

İndice H: 3

Orcid ID: https://orcid.org/0000-0001-9129-5673

Google Scholar: https://scholar.google.com.sg/citations?user=UsVJTKUAAAAJ\&hl=th 